\title{
Three-dimensional relationship between the critical contact angle and the torque angle
}

\author{
Bo-Sun Kang, DDS, MSD, PhD, ${ }^{a}$ Seung-Hak Baek, DDS, MSD, PhD, ${ }^{\mathrm{b}}$ James Mah, DDS, MSD, PhD, ${ }^{\mathrm{c}}$ and \\ Won-Sik Yang, DDS, MSD, PhD ${ }^{\mathbf{d}}$ \\ Seoul, Korea, and Los Angeles, Calif
}

The purpose of this study was to analyze the relationship between the critical contact angle and the torque angle in an orthodontic bracket and archwire assembly in 3 dimensions. Three-dimensional mathematical models were created with geometric bracket-archwire parameters that included 2 slot sizes, 3 bracket widths, and 3 to 4 wire sizes. From this, 3-dimensional mathematical equations (3DMEs) for the critical contact angle and the maximum torque that result in critical contact angles of 0 were derived and calculated. To evaluate the effects of archwire-bracket parameters on critical contact angles, analysis of variance (ANOVA) was performed at the significance level of $P \leq .05$. For all bracket-archwire combinations, the critical contact angle decreased as bracket width, torque angle, and wire size increased. Therefore, all bracket-archwire parameters except slot height had an effect on the critical contact angle. Results of the critical contact angle produced from our 3DMEs were the same as those produced by 3D computer-aided design (SolidWorks Corp, Concord, Mass), thus confirming the validity of our derived equations. In addition, the effect of a beveled edge was investigated in some archwires. Furthermore, torsional play angles were calculated and found to be similar to those in previous reports. The results of this study provide theoretic and experimental bases for clinical orthodontic practice and indicate that torque angles should be included in the evaluation of the critical contact angle. (Am J Orthod Dentofacial Orthop 2003;123:64-73)

$\mathbf{S}$ liding mechanics are commonly used in orthodontics to open or close space in the dental arch. In fact, most techniques of fixed appliances involve some sliding between the archwire and the bracket. Whenever sliding occurs, frictional resistance is encountered. ${ }^{1}$ Clinicians need to understand the impact of frictional resistance between the brackets and the archwires to design and implement biomechanics for optimal tooth movement and biologic tissue response. It is essential to control resistance to sliding to provide more efficient and predictable results and thereby to increase the quality of treatment. Resistance to sliding is a combination of classic friction, archwirebracket binding, and archwire notching. ${ }^{2}$

The angle at which the clearance between the

\footnotetext{
aPrivate practice, Seoul, Korea.

${ }^{\mathrm{b}}$ Assistant Professor, Department of Orthodontics, College of Dentistry, Seoul National University, Seoul, Korea.

${ }^{\mathrm{c} A s s i s t a n t}$ Professor, Division of Craniofacial Sciences and Therapeutics, School of Dentistry, University of Southern California, Los Angeles.

${ }^{\mathrm{d}}$ Professor Emeritus, Department of Orthodontics, College of Dentistry, Seoul National University, Seoul, Korea.

Reprint requests to: Seung-Hak Baek, DDS, MSD, PhD, Department of Orthodontics, College of Dentistry, Seoul National University, \#28 Yeonkundong, Jongro-ku, Seoul, 110-749, South Korea; e-mail, drwhite@unitel.co.kr. Submitted, December 2001; revised and accepted, April 2002.

Copyright (C) 2003 by the American Association of Orthodontists.

$0889-5406 / 2003 / \$ 30.00+0$

doi: $10.1067 / \bmod .2003 .55$
}

archwire and the bracket first disappears is defined as the critical contact angle $\left(\theta_{\mathrm{c}}\right)^{3}$ If the angulation between the archwire and the bracket slot is less than the critical contact angle in the passive configuration, only classic friction is important, because binding ${ }^{4,5}$ and notching ${ }^{6}$ are nonexistent (Fig 1, $A$ ). Several investigators have studied the causes and effects of resistance to sliding in the passive configuration as a function of wire alloy, bracket material, surface modification and roughness, and wet and dry states. ${ }^{1,7-24}$

As the angulation between the bracket and the archwire increases, the clearance between the archwire and the bracket slot disappears, and an interference fit occurs. Therefore, binding arises as a second component of resistance to sliding. ${ }^{3,25}$ This condition is referred to as the active configuration (Fig 1,B). Resistance to sliding increases as much as 100 -fold when archwire-bracket couples exist in the active configuration. ${ }^{25}$ This is more correctly attributable to binding rather than to true friction. ${ }^{8}$ However, the active configuration has received considerably less attention, primarily because of the experimental difficulties associated with measuring $\theta$ at angles greater than $0^{\circ} .{ }^{2}$ In addition, there are few mathematical approaches that relate the specific dimensions of archwire-bracket couples and their angulations to sliding performance. $^{3}$ 
(a)

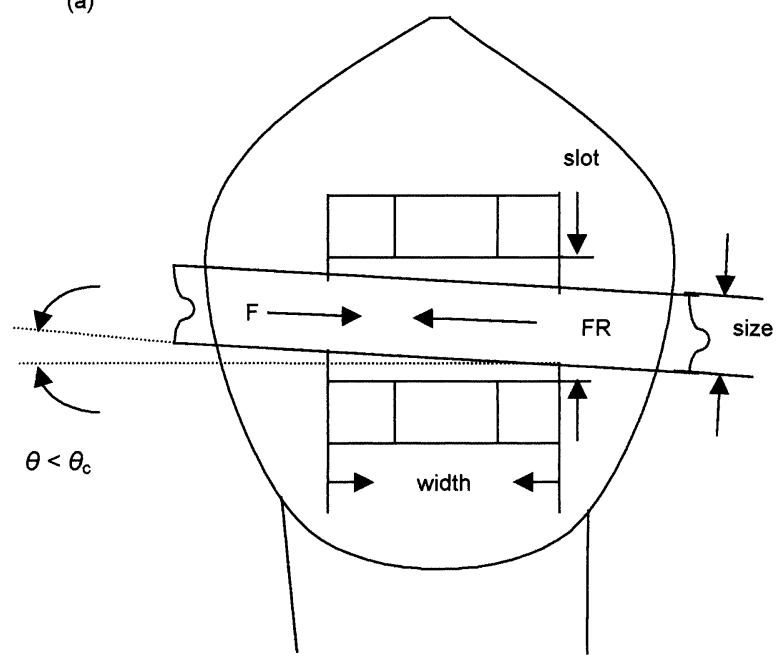

(b)

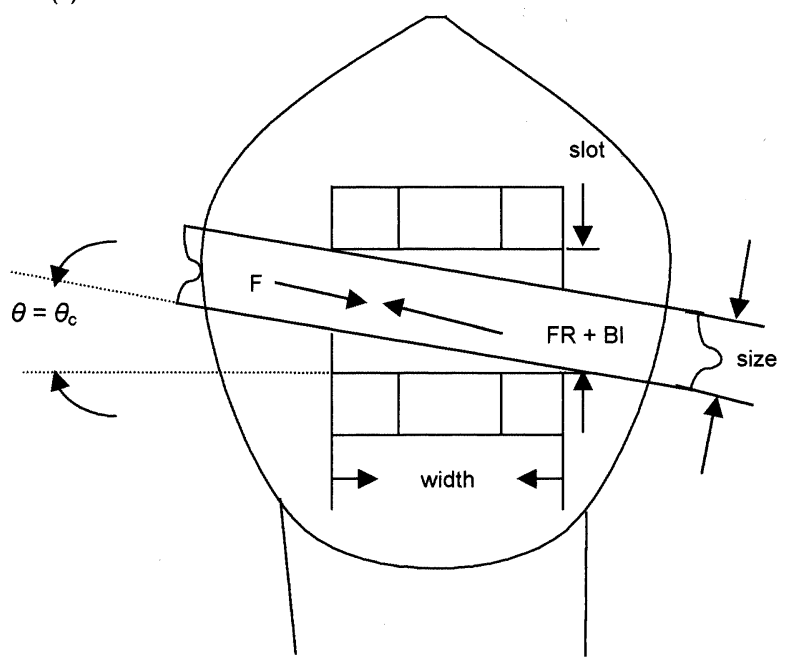

Fig 1. Passive and active configuration in 2 dimensions. A, In passive configuration, angulation ( $\theta)$ is less than the critical contact angle of interference fit $\left(\theta_{c}\right)$. The applied force $(F)$ is opposed only by classic friction $(F R)$. Note 3 important geometric terms associated with archwire-bracket couple: size (archwire size), slot (bracket slot size), and width (bracket width). B, In active configuration, $F$ is opposed by binding $(B I)$ in addition to $F R$ because of contact with bracket slot when $\theta=\theta_{c}$.

Kusy and Whitley ${ }^{2}$ described a 2-dimensional theory formed on the basis of relative geometry of the archwire-bracket couple. The critical contact angle and the second-order clearance were described by 3 geometric parameters: the dimension of the archwire that engages the floor of the slot (size), the corresponding bracket dimension at the floor of the slot (slot), and the mesiodistal width of the bracket (width) (Fig 1). Kusy and Whitley ${ }^{2,3}$ calculated the critical contact angle without torque (Equ. 1).

This theory deals only with the first and second orders of space.

The torque factor that is fundamental to 3-dimensional (3D) control of edgewise appliances was not included. However, a knowledge of torque control is vital to appliance design and force delivery. Clinicians must understand the intricate role of torque relationships in the different stages of fixed appliance therapy.

Therefore, the present study was conducted to express the 3D relationship of the critical contact angle under varying torque conditions in a 3-dimensional mathematical equation (3DME). The 3DME was validated by comparing the calculated critical contact angles and torsional play angles with those produced by a 3D computer-aided design (CAD) system and with previous calculations. ${ }^{26}$

\section{MATERIAL AND METHODS \\ Derivation of 3DME and calculation of critical contact angle and maximum torque angle}

We established a 3D mathematical model by using 3D geometric archwire-bracket parameters such as 2 slot sizes, 3 bracket widths, and 3 to 4 wire sizes in each slot (Table I). 3DMEs were derived, and the critical contact angles between the brackets and the archwires as a function of torque angle were calculated. The torque angle began at $0^{\circ}$ and proceeded in increments of $1^{\circ}$ until the critical contact angles came to near $0^{\circ}$. Also, the maximum torque angles that result in critical contact angles of 0 were calculated. The changes in the critical contact angles were also calculated. To evaluate the effects of the archwire-bracket parameters on the critical contact angles and the torque angles, analysis of variance (ANOVA) was performed at the significance level of $P \leq .05$.

Equ. 1. $\quad \cos \theta_{c}=\frac{\operatorname{size}^{2}-\text { width }^{2}}{(\text { size })(\text { slot }) \pm \sqrt{(\text { width })^{2}\left[-(\text { size })^{2}+(\text { slot })^{2}+(\text { width })^{2}\right]}}$ 
Table I. Critical contact angles of bracket width-archwire size combinations as function of torque angle

\begin{tabular}{|c|c|c|c|c|c|c|c|c|c|c|c|c|c|c|c|}
\hline \multirow{2}{*}{$\begin{array}{l}\text { Bracket } \\
\text { slot (in) }\end{array}$} & \multirow{2}{*}{$\begin{array}{c}\text { Bracket } \\
\text { width }(\mathrm{mm})\end{array}$} & \multirow{2}{*}{$\begin{array}{l}\text { Archwire size } \\
\text { (in) }\end{array}$} & \multicolumn{13}{|c|}{ Torque angle $\left({ }^{\circ}\right)$} \\
\hline & & & 0 & 1 & 2 & 3 & 4 & 5 & 6 & 7 & 8 & 9 & 10 & 11 & 12 \\
\hline \multirow[t]{9}{*}{$.018 \times .025$} & \multirow[t]{3}{*}{2.5} & $.016 \times .016^{\mathrm{a}}$ & 1.16 & 1.00 & 0.84 & 0.69 & 0.54 & 0.39 & \multirow[t]{3}{*}{0.24} & \multirow[t]{3}{*}{0.10} & & & & & \\
\hline & & $.016 \times .022^{\mathrm{b}}$ & 1.16 & 0.94 & 0.72 & 0.51 & 0.29 & 0.08 & & & & & & & \\
\hline & & $.017 \times .025^{\mathrm{c}}$ & 0.58 & 0.33 & 0.08 & & & & & & & & & & \\
\hline & \multirow[t]{3}{*}{3.5} & $.016 \times .016^{\mathrm{a}}$ & 0.83 & 0.72 & 0.60 & 0.49 & 0.38 & 0.28 & \multirow[t]{3}{*}{0.17} & \multirow[t]{3}{*}{0.07} & & & & & \\
\hline & & $.016 \times .022 * \mathrm{~b}$ & 0.83 & 0.67 & 0.52 & 0.36 & 0.21 & 0.06 & & & & & & & \\
\hline & & $.017 \times .025^{\mathrm{c}}$ & 0.42 & 0.24 & 0.06 & & & & & & & & & & \\
\hline & \multirow[t]{3}{*}{4.5} & $.016 \times .016^{\mathrm{a}}$ & 0.65 & 0.56 & 0.47 & 0.38 & 0.30 & 0.22 & \multirow[t]{3}{*}{0.14} & \multirow[t]{3}{*}{0.06} & & & & & \\
\hline & & $.016 \times .022^{\mathrm{b}}$ & 0.65 & 0.52 & 0.40 & 0.28 & 0.16 & 0.05 & & & & & & & \\
\hline & & $.017 \times .025^{\mathrm{c}}$ & 0.32 & 0.18 & 0.04 & & & & & & & & & & \\
\hline \multirow[t]{12}{*}{$.022 \times .028$} & \multirow[t]{4}{*}{2.5} & $.017 \times .025^{\mathrm{d}}$ & 2.90 & 2.65 & 2.40 & 2.16 & 1.92 & 1.68 & 1.45 & 1.22 & 0.99 & 0.76 & \multirow[t]{4}{*}{0.54} & \multirow[t]{4}{*}{0.32} & \multirow[t]{4}{*}{0.10} \\
\hline & & $.018 \times .025^{\mathrm{e}}$ & 2.32 & 2.07 & 1.82 & 1.58 & 1.34 & 1.10 & 0.87 & 0.64 & 0.41 & 0.18 & & & \\
\hline & & $.019 \times .025^{\mathrm{f}}$ & 1.74 & 1.49 & 1.24 & 1.00 & 0.76 & 0.52 & 0.29 & 0.06 & & & & & \\
\hline & & $.021 \times .025^{\mathrm{g}}$ & 0.58 & 0.33 & 0.08 & & & & & & & & & & \\
\hline & \multirow[t]{4}{*}{3.5} & $.017 \times .025^{\mathrm{d}}$ & 2.07 & 1.89 & 1.72 & 1.54 & 1.37 & 1.20 & 1.04 & 0.87 & 0.71 & 0.55 & \multirow[t]{4}{*}{0.39} & \multirow[t]{4}{*}{0.23} & \multirow[t]{4}{*}{0.07} \\
\hline & & $.018 \times .025^{\mathrm{e}}$ & 1.66 & 1.48 & 1.30 & 1.13 & 0.96 & 0.79 & 0.62 & 0.46 & 0.29 & 0.13 & & & \\
\hline & & $.019 \times .025^{* \mathrm{f}}$ & 1.25 & 1.07 & 0.89 & 0.71 & 0.54 & 0.37 & 0.21 & 0.04 & & & & & \\
\hline & & $.021 \times .025^{\mathrm{g}}$ & 0.42 & 0.24 & 0.06 & & & & & & & & & & \\
\hline & \multirow[t]{4}{*}{4.5} & $.017 \times .025^{\mathrm{d}}$ & 1.61 & 1.47 & 1.34 & 1.20 & 1.07 & 0.94 & 0.81 & 0.68 & 0.55 & 0.42 & \multirow[t]{4}{*}{0.30} & \multirow[t]{4}{*}{0.18} & \multirow[t]{4}{*}{0.06} \\
\hline & & $.018 \times .025^{\mathrm{e}}$ & 1.29 & 1.15 & 1.01 & 0.88 & 0.75 & 0.61 & 0.48 & 0.35 & 0.23 & 0.10 & & & \\
\hline & & $.019 \times .025^{\mathrm{f}}$ & 0.97 & 0.83 & 0.69 & 0.56 & 0.42 & 0.29 & 0.16 & 0.03 & & & & & \\
\hline & & $.021 \times .025^{\mathrm{g}}$ & 0.32 & 0.18 & 0.05 & & & & & & & & & & \\
\hline
\end{tabular}

*Accuracy of critical torque angle was verified with 3D CAD. Empty cells indicate maximum torque angle has been reached.

ANOVA test showed all values of archwire size-bracket width combinations $\left({ }^{a, b, d, e, f}\right)$ were significant at $\mathrm{P}<.05$ except $.017 \times .025$ in archwire-bracket width in $.018 \times .025$-in bracket slot $\left({ }^{\mathrm{c}}\right)$ and $.021 \times .025$-in archwire-bracket width in $.022 \times .028$-in bracket slot $\left({ }^{\mathrm{g}}\right)$.

\section{CAD analysis}

To verify the accuracy of the critical contact angles obtained from the 3DMEs, simulation of the bracketarchwire assembly (SolidWorks 2000, SolidWorks Corp, Concord, Mass), measurement of torsional displacement of the archwire within the bracket (Designspace 5.0, ANSYS Inc Corp, Canonsburg, Pa), and 3D display with the use of 3D CAD software (PhotoWorks 2000, SolidWorks Corp) were performed.

Two bracket-archwire combinations commonly used for orthodontic treatment-a $.016 \times .022$-in archwire in an $.018 \times .025$-in bracket (width, $3.5 \mathrm{~mm}$ ) and a $.019 \times .025$-in archwire in a $.022 \times .028$-in bracket (width, $3.5 \mathrm{~mm}$ )—were modeled.

The critical contact angle of archwires with beveled edges was calculated and compared with those measured by the 3D CAD system. In addition, we compared the maximum torque angles that result in critical contact angles of 0 between both systems. Torsional play was calculated according to the formula of Meling et al. ${ }^{26}$

\section{RESULTS}

\section{DMEs for critical contact angle and maximum torque angle}

To include torque, we created a 3D space where the $\mathrm{z}$-axis denotes height, the $\mathrm{x}$-axis denotes width, and the $y$-axis denotes length. A computer model was produced with an archwire with width (B), height (A), and length (L) sufficient for manipulation or rotation (Fig 2, A). Torque is defined as the angle that rotates around the $y$-axis. After a specific amount of torque around the length of the archwire is applied (Fig 2,B), it will bind in the bracket (Fig 2,C). During this action, the critical contact angle is established because it depends on the sizes of the archwire and the bracket. The derived 3DME for the critical contact angle is given in Equation 2 (see Appendix I):

$$
\text { Equ. 2. } \quad \cos \theta_{c}=\frac{\mathrm{A}^{2} \cos ^{2} \phi-\mathrm{b}^{2} \cos ^{2} \phi}{\mathrm{A} \cos \phi(\mathrm{a}-\mathrm{B} \sin \phi) \pm \sqrt{\mathrm{b}^{2} \cos ^{2} \phi\left(-(\mathrm{A} \cos \phi)^{2}+(\mathrm{a}-\mathrm{B} \sin \phi)^{2}+(\mathrm{b} \cos \phi)^{2}\right.}}
$$


(a)

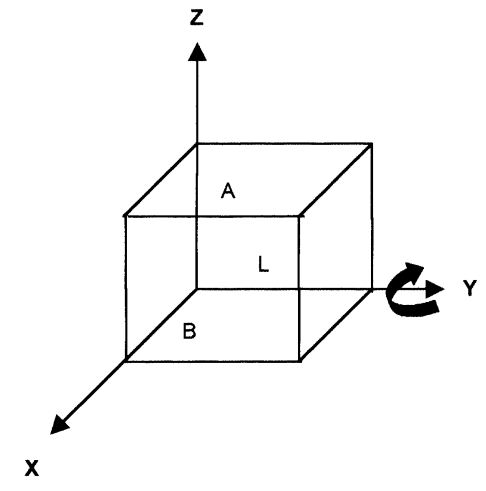

(b)

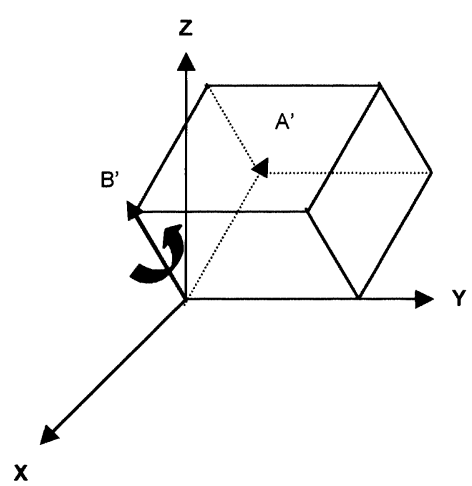

(c)

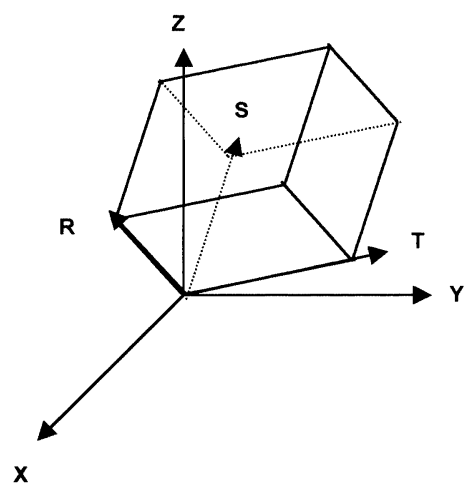

Fig 2. Derivation of 3DME. A, Model and notations: $A=<0,0, \mathrm{~A}>\mathrm{B}=<\mathrm{B}, 0,0>\mathrm{L}=<0, \mathrm{~L}, 0>$. $A$, archwire height; $B$, archwire width; $L$, archwire length. $\mathbf{B}$, Result of rotation around $y$-axis for torque. Vectors $B$ and $A$ are changed to vectors $B^{\prime}$ and $A^{\prime}$, respectively. C, Result of rotation around vector $B^{\prime}$ for binding. Vectors $B^{\prime}, A^{\prime}$, and $L$ are changed to vectors $R, S$, and $T$, respectively.

where $a$ is bracket slot size, $b$ is bracket width, $A$ is archwire height, $B$ is archwire width, $\theta$ is critical contact angle, and $\phi$ is torque angle.

The derived 3DME for the maximum torque angle that results in a critical contact angle of 0 with the given archwire and orthodontic bracket is as follows (see Appendix II):

$$
\cos \phi=\frac{\mathrm{a}^{2}-\mathrm{B}^{2}}{\mathrm{Aa} \pm \sqrt{\mathrm{B}^{2}\left(-\mathrm{a}^{2}+\mathrm{A}^{2}+\mathrm{B}^{2}\right)}}
$$

where $a$ is bracket slot size, $A$ is archwire height, $B$ is archwire width, and $\phi$ is torque angle.

\section{Calculations of critical contact angles}

The critical contact angles were calculated in an $.018 \times .025$-in bracket for 3 wires and in a $.022 \times$ .028-in bracket for 4 wires as a function of torque angle with the 3DME referred to earlier (Table I). For all bracket-archwire combinations, the critical contact angle decreased as bracket width, torque angle, and wire size increased (Table I). The mean changes of the critical contact angles increased as did wire size. The equation also showed that slot height had no effect on the critical contact angle.

ANOVA showed significant differences in the critical contact angles of the bracket width-archwire combinations at each torque angulation $(P<.05)$ except for the $.017 \times .025$-in archwire in the $.022 \times .028$-in bracket and the $.021 \times .025$-in archwire in the $.022 \times$ .028-in bracket (Table I).

Changes in the critical contact angle increased with increases in archwire size (Table II). However, as the bracket width increased, the amount of change in the critical contact angle lessened (Table II).

Significant differences were found in the changes in the critical contact angle of almost all bracket-archwire combinations as a function of torque angle between $.018 \times .025$-in and $.022 \times .028$-in brackets (Table II). However, intragroup comparisons of the 2.5-, 3.5-, and 4.5-mm groups with the 4 different archwire sizes in the $.022 \times .028$-in bracket showed no significant differences in the changes in critical contact angles with different archwires (Table II).

The maximum torque angles, which result in critical contact angles of 0 , decreased with increases in archwire size (Table III). Bracket width had no effect on this calculation.

\section{CAD analysis}

The amount of torsional displacement of the archwire within the bracket was examined by 3D CAD analysis (Fig 3,A). Deformation of the archwire within the bracket can be seen when structural forces are placed only at both ends of the bracket (Fig 3, B). The amount of the torsional displacement was insignificant (data not shown).

In a comparison with the critical contact angle and the maximum torque angle, the $3 \mathrm{DME}$ and the nonbeveled edge showed almost no difference (Table IV). When archwires with beveled edges were examined, the maximum torque angle increased by approximately $1^{\circ}$ over nonbeveled archwires modeled by CAD and the 3DME (Table IV). Changes in the critical contact angle of the archwire and bracket combinations examined 
Table II. Changes in critical contact angles of archwire size-bracket width combinations in $.018 \times .025$-in and $.022 \times .028$-in bracket slot

\begin{tabular}{|c|c|c|c|c|c|c|c|}
\hline \multirow{3}{*}{$\begin{array}{l}\text { Bracket slot } \\
\text { (in) }\end{array}$} & \multirow{3}{*}{$\begin{array}{l}\text { Archwire size } \\
\text { (in) }\end{array}$} & \multicolumn{6}{|c|}{ Bracket width (mm) } \\
\hline & & \multicolumn{2}{|c|}{2.5} & \multicolumn{2}{|c|}{3.5} & \multicolumn{2}{|c|}{4.5} \\
\hline & & Mean & $S D$ & Mean & $S D$ & Mean & $S D$ \\
\hline \multirow[t]{3}{*}{$.018 \times .025$} & $.016 \times .016$ & -0.15 & 0.006 & -0.11 & 0.004 & -0.08 & 0.003 \\
\hline & $.016 \times .022$ & -0.22 & 0.004 & -0.15 & 0.003 & -0.12 & 0.002 \\
\hline & $.017 \times .025$ & -0.25 & 0.002 & -0.18 & 0.001 & -0.14 & 0.001 \\
\hline \multirow[t]{4}{*}{$.022 \times .028$} & $.017 \times .025$ & $-0.23^{\mathrm{a}}$ & 0.010 & $-0.17^{\mathrm{b}}$ & 0.008 & $-0.13^{\mathrm{c}}$ & 0.006 \\
\hline & $.018 \times .025$ & $-0.24^{\mathrm{a}}$ & 0.008 & $-0.17^{\mathrm{b}}$ & 0.006 & $-0.13^{\mathrm{c}}$ & 0.005 \\
\hline & $.019 \times .025$ & $-0.24^{\mathrm{a}}$ & 0.007 & $-0.17^{\mathrm{b}}$ & 0.005 & $-0.13^{\mathrm{c}}$ & 0.004 \\
\hline & $.021 \times .025$ & $-0.25^{\mathrm{a}}$ & 0.002 & $-0.18^{\mathrm{b}}$ & 0.002 & $-0.14^{\mathrm{c}}$ & 0.001 \\
\hline
\end{tabular}

ANOVA test showed all values of archwire size-bracket width combinations were significant at $P<.05$ except intragroup archwire size-bracket width combinations in $.022 \times .028$-in bracket slot $\left({ }^{\mathrm{a}, \mathrm{b}, \mathrm{c}}\right)$.

Table III. Maximum torque angles that result in critical contact angle of 0

\begin{tabular}{lcc}
\hline $\begin{array}{l}\text { Bracket slot* } \\
\text { (in) }\end{array}$ & $\begin{array}{c}\text { Archwire size } \\
\text { (in) })\end{array}$ & $\begin{array}{c}\text { Maximum } \\
\text { torque angle }\left({ }^{\circ}\right)\end{array}$ \\
\hline $.018 \times .025$ & $.016 \times .016$ & 7.70 \\
& $.016 \times .022$ & 5.40 \\
& $.017 \times .025$ & 2.32 \\
$.022 \times .028$ & $.017 \times .025$ & 12.48 \\
& $.018 \times .025$ & 9.82 \\
& $.019 \times .025$ & 7.24 \\
& $.021 \times .025$ & 2.33 \\
\hline
\end{tabular}

*Bracket width had no effect on this calculation

were not significant (Table IV). These values were almost the same as those produced with the formula of Meling et al, ${ }^{26}$ corroborating our methods (Table IV).

\section{DISCUSSION}

Static and kinetic friction are parameters that concern a practitioner who uses sliding mechanics. Other important parameters are binding and notching effects that arise after an interference fit has occurred and sliding has ceased. ${ }^{25}$ When the orthodontic wire and the bracket are in the passive configuration, the archwirebracket combinations have the least amount of frictional force. ${ }^{27}$ However, in the active configuration, brackets are subjected to higher levels of stress. Binding is described as the point at which an interference fit occurs between the archwire and the bracket at a particular value of $\theta .{ }^{17}$ As an immediate consequence, resistance to sliding increases because of this phenomenon. Although binding has always been included in the measurement of resistance to sliding, binding has never been formalized.

Recently, the critical contact angle was calculated as the boundary between classic frictional behavior and binding-related phenomena. ${ }^{2}$ The 3DME from this study incorporates wire-bracket geometry and torque angle into the analysis of the active configuration. This mathematical equation can be used to compare different wires and brackets on the basis of critical contact angles. Because most of these variables are under the control of the clinician, careful selection of appropriate brackets, wires, and torque angles can help to achieve optimal control of tooth movement and anchorage.

\section{Effects of nominal bracket-archwire parameters as a function of torque angle on critical contact angle}

When torque angles were 0 , our results were in accordance with the findings of Kusy and Whitley ${ }^{2,3}$ (Table I). The archwire width had no effect on the critical contact angles when the torque angles were 0 . However, as the torque angle increased, the archwire width had an effect on the critical contact angle as a function of torque angle. In addition, as the torque angle increased, the critical contact angles decreased for all archwire-bracket combinations. These results show that the critical contact angle decreases in a nonlinear manner with increased torque angulation.

As the archwire size increased, the critical contact angles decreased. For example, the critical contact angle $\left(\theta_{\mathrm{c}}\right)$ of a $.018 \times .025$-in bracket slot with a 3.5 -mm bracket width (Fig 4) and of a $.022 \times .028$-in bracket slot with a $3.5-\mathrm{mm}$ bracket width (Fig 5) decreased according to increases in archwire size. This is consistent with previous investigations on bracketarchwire binding that found that, as wire size increases, so does the frictional force between the bracket and the archwire. $^{4}$

For all bracket-archwire combinations in a $.018 \times$ .025-in bracket, there were significant differences in the changes in the critical contact angle as a function of 


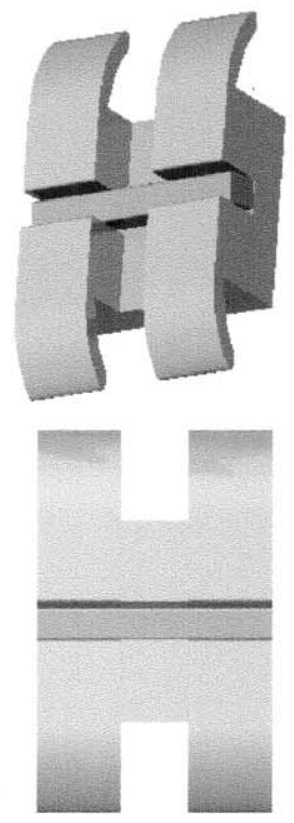

a)
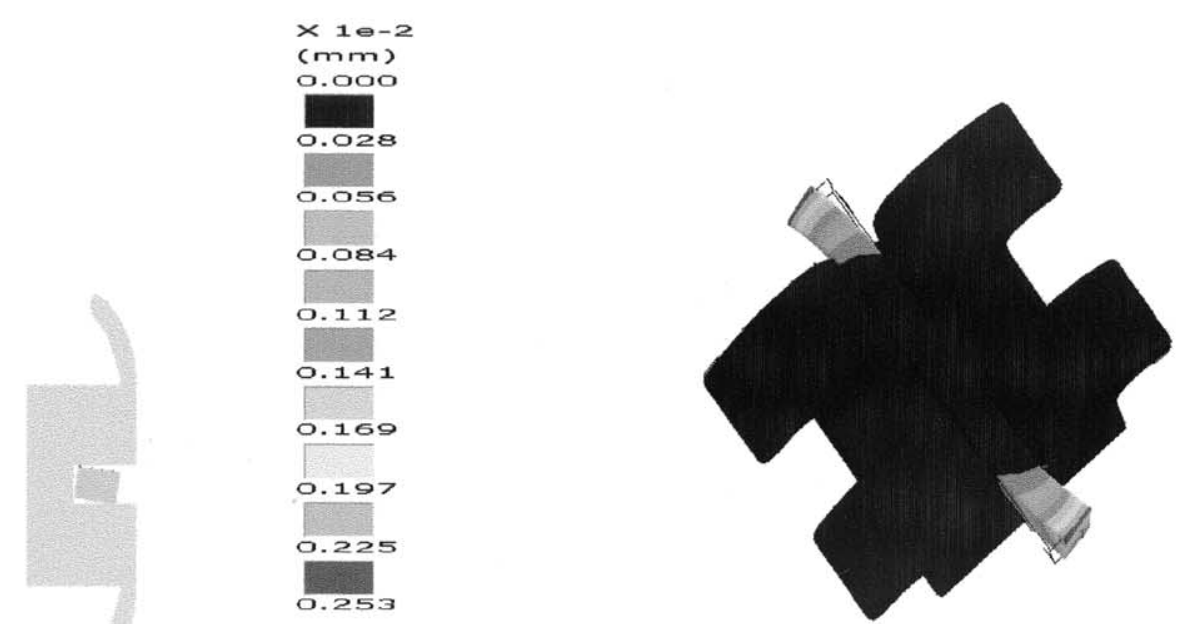

Fig 3. $3 D$ CAD analysis. A, CAD model (bracket-archwire assembly) used in this study. B, Torsional displacement of archwire within bracket with use of mechanical design analysis program.

Table IV. Comparison of critical contact angles and maximum torque angles that result in critical contact angle of 0 between 3DME and CAD model with beveled edge effect

\begin{tabular}{|c|c|c|c|c|c|c|c|c|c|c|c|c|}
\hline \multirow{2}{*}{$\begin{array}{l}\text { Bracket slot, } \\
\text { bracket width, } \\
\text { and archwire } \\
\text { size }\end{array}$} & \multicolumn{10}{|c|}{ Critical contact angle $\left({ }^{\circ}\right)$} & \multirow{2}{*}{$\begin{array}{c}\text { Maximum } \\
\text { torque angle } \\
\left({ }^{\circ}\right)\end{array}$} & \multirow{2}{*}{$\begin{array}{c}\text { Equation of } \\
\text { Meling et } \mathrm{al}^{26} \\
\left({ }^{\circ}\right)\end{array}$} \\
\hline & Torque angle & 0 & 1 & 2 & 3 & 4 & 5 & 6 & 7 & 8 & & \\
\hline $.018 \times .025$-in & 3DME & 0.83 & 0.67 & 0.52 & 0.36 & 0.21 & 0.06 & $\mathrm{na}^{1}$ & $\mathrm{na}^{1}$ & $\mathrm{na}^{1}$ & 5.40 & $\mathrm{na}^{2}$ \\
\hline $3.5 \mathrm{~mm}$ & CAD (nonbeveled edge) & 0.83 & 0.67 & 0.52 & 0.36 & 0.21 & 0.06 & $\mathrm{na}^{1}$ & $\mathrm{na}^{1}$ & $\mathrm{na}^{1}$ & 5.26 & $\mathrm{na}^{2}$ \\
\hline $.016 \times .022$-in & CAD (beveled edge) & 0.83 & 0.70 & 0.56 & 0.43 & 0.30 & 0.17 & 0.04 & $\mathrm{na}^{1}$ & $\mathrm{na}^{1}$ & 6.24 & $6.33^{*}$ \\
\hline $.022 \times .028$-in & 3DME & 1.25 & 1.07 & 0.89 & 0.71 & 0.54 & 0.37 & 0.21 & 0.04 & $\mathrm{na}^{1}$ & 7.24 & $\mathrm{na}^{2}$ \\
\hline $3.5 \mathrm{~mm}$ & CAD (nonbeveled edge) & 1.25 & 1.07 & 0.89 & 0.71 & 0.54 & 0.37 & 0.20 & 0.004 & $\mathrm{na}^{1}$ & 7.26 & $\mathrm{na}^{2}$ \\
\hline $.019 \times .025$-in & CAD (beveled edge) & 1.25 & 1.09 & 0.93 & 0.78 & 0.63 & 0.48 & 0.33 & 0.19 & 0.005 & 8.29 & $8.33 *$ \\
\hline
\end{tabular}

*Accuracy of maximum torque angle was verified with formula of Meling et al. ${ }^{26}$ in $.016 \times .022$-in archwire in $.018 \times .025$-in bracket slot $(3.5$ $\mathrm{nm}$ bracket width) and $.019 \times .025$-in archwire in $.022 \times .028$-in bracket slot $(3.5 \mathrm{~mm}$ bracket width).

$n a^{l}$, not applicable because torque angle cannot exceed maximum torque angle.

$n a^{2}$, does not apply.

torque angle $(P<.05)$ (Table II). However, for the bracket-archwire combinations in a .022 $\times .028$-in bracket, there were no significant differences in the changes in the critical contact angles between different archwire size groups (Table II). However, the changes in the critical contact angle increased with increases in archwire size for both $.018 \times .025$-in and $.022 \times$ .028-in bracket slots (Table II). For all bracket-archwire combinations, the changes in critical contact angle decreased with increases in torque angles. From these results, we found that as the torque increased, the effect of torque angle on the critical contact decreased.

The critical contact angle decreased as the bracket width increased from 2.5 to $4.5 \mathrm{~mm}$ (Table I). For bracket width, the critical contact angle was relatively small for a $.017 \times .025$-in wire in a bracket with a $.018 \times .025$-in slot $($ Table I) and a $.021 \times .025$-in wire in a bracket with a $.022 \times .028$-in slot $($ Table I). There 


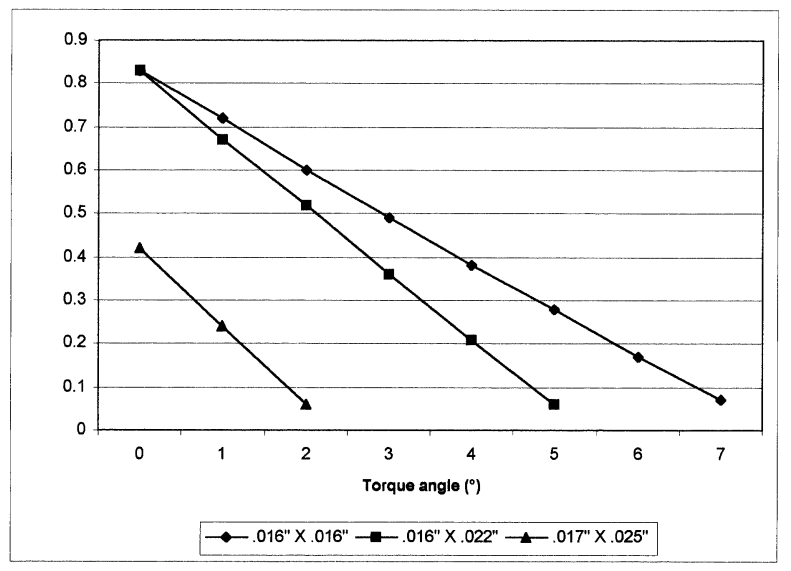

Fig 4. Change of critical contact angle $\left(\theta_{c}\right)$ according to archwire size in $.018 \times .025$-in bracket slot and 3.5- $\mathrm{mm}$ bracket width.

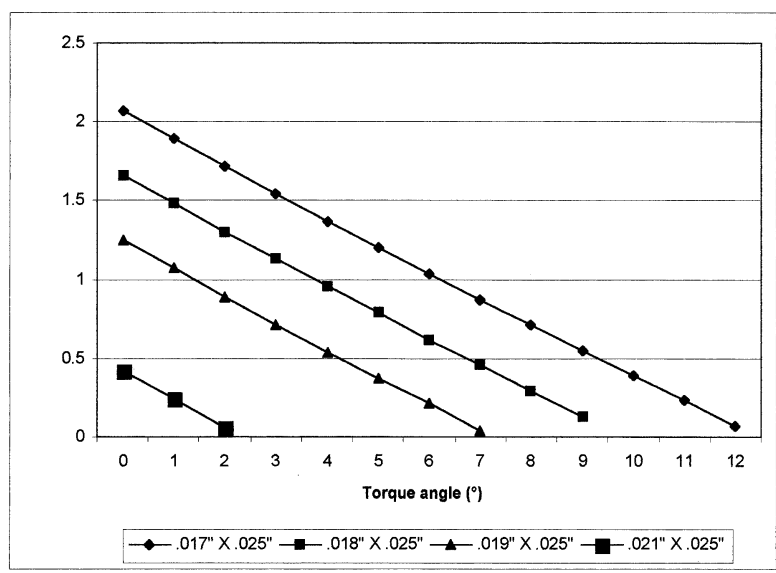

Fig 5. Change of critical contact angle $\left(\theta_{c}\right)$ according to archwire size in $.022 \times .028$-in bracket slot and 3.5- $\mathrm{mm}$ bracket width.

were no significant differences between these bracket width groups (Table I), but all other bracket-archwire combinations showed significant differences between the critical contact angles of the different width groups (Table I). As the bracket width increased, the changes in the critical contact angles decreased. For example, the critical contact angles $\left(\theta_{c}\right)$ of a $.016 \times .022$-in archwire and a $.018 \times .025$-in bracket (Fig 6) and of a $.019 \times .025$-in archwire and a $.022 \times .028$-in bracket (Fig 7) decreased according to increases in bracket width.

For a $.018 \times .025$-in slot, the maximum torque angles that produced critical contact angles of 0 were from $2.32^{\circ}$ to $7.70^{\circ}$ (Table III). For a $.022 \times .028$-in slot, the maximum torque angles that produced a

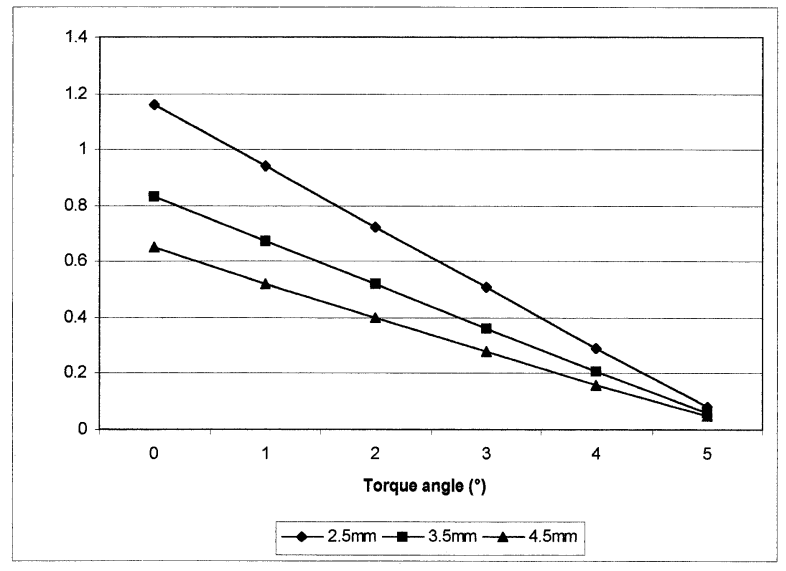

Fig 6. Change of the critical contact angle $\left(\theta_{c}\right)$ according to bracket width in $.016 \times .022$-in archwire and $.018 \times .025$-in bracket slot.

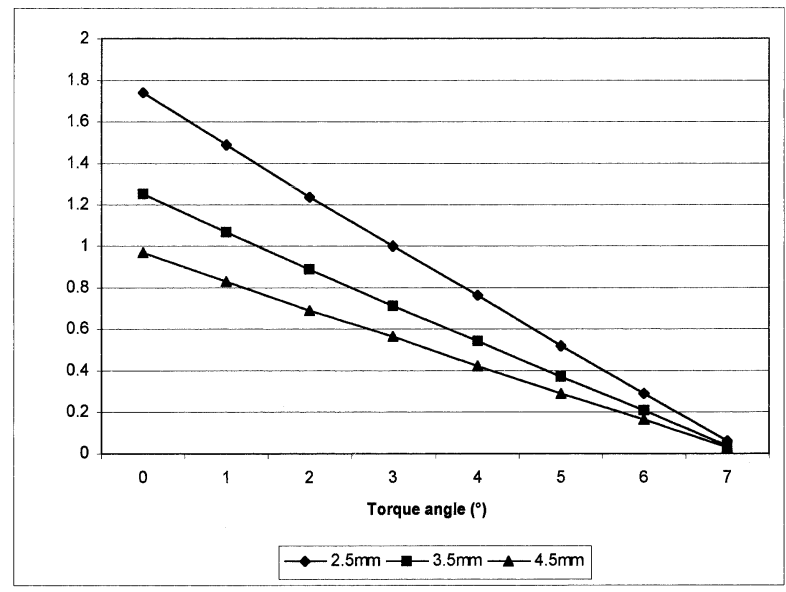

Fig 7. Change of critical contact angle $\left(\theta_{c}\right)$ according to bracket width in $.019 \times .025$-in archwire and $.022 \times$ .028-in bracket slot.

critical contact angle of 0 were from $2.33^{\circ}$ to $12.48^{\circ}$ (Table III). For a $.017 \times .025$-in archwire, the maximum torque angle of a $.022 \times .028$-in slot was 5.38 times as large as that of a $.018 \times .025$-in slot (Table III). For a $.019 \times .025$-in archwire in a $.022 \times .028$-in slot, the maximum torque angle was $7.24^{\circ}$. This suggests that if this archwire-bracket combination is used to produce torque on a tooth, the amount of torque applied to the archwire should be more than $7.24^{\circ}$. In addition, these results suggest that torque should be established before using sliding mechanics. Bracket width had no effect on the maximum torque angle that results in a critical contact angle of 0 (Table III).

For all bracket-archwire combinations, the critical 
contact angle decreased as bracket width, torque angle, and wire size increased (Table I). Therefore, all bracket-archwire parameters except slot height had an effect on the critical contact angle.

\section{CAD data analysis}

In orthodontic treatment in which arch-guided tooth movement is used, rectangular archwires control 3D tooth movement. These archwires contain beveled edges to optimize sliding mechanics and to improve patient comfort. $^{28}$ It has been suggested that the bevel radius of the edge should be at least $0.04 \mathrm{~mm}(0.0016$ in) for patient comfort. ${ }^{29,30}$ Therefore, we included archwires with and without beveled edges $(0.04-\mathrm{mm}$ radius) in the CAD model.

We used our 3DMEs and the formula of Meling et $\mathrm{al}^{26}$ (Table IV) to calculate the torsional play and found agreement with the theoretic calculations. The maximum torque angles that resulted in a critical contact angle of 0 were very similar for 3DMEs and nonbeveled-edge CAD (Table IV). These data confirm the accuracy of the derived 3DME. When the edge bevels were applied in the CAD model archwire, the difference was about $1^{\circ}$. The formula of Meling et $\mathrm{al}^{26}$ (Table IV) also accounts for edge bevel-corner radius, and the results compare favorably with those of the CAD model with the beveled edge. If the edge rounding and the dimensional deviations are taken into account, ${ }^{31}$ actual torsional play can be much greater than theoretic torsional play.

The results of this study provide some theoretic and experimental basis for clinical practice. One essential component of successful sliding mechanics is the precise calculation of the critical contact angle between the brackets and the archwires. The amount of applied force lost because of resistance to sliding can range from $12 \%$ to $60 \%{ }^{17}$ This result might explain some of the variability of fixed appliances. If 1 of the causes of resistance to sliding is better managed by using these results, clinical practitioners can improve the efficiency of fixed appliances.

\section{CONCLUSIONS}

To investigate the critical contact angle between the orthodontic bracket and the archwire mathematically (3D analysis including torque factor), a 3D mathematical model was established, and theoretic equations were derived so that precise critical contact angles could be determined in terms of the nominal archwirebracket parameters as a function of torque angle. The accuracy of the mathematical equation and the importance of torsional play was confirmed with a CAD system.
For all bracket-archwire combinations, the critical contact angle decreased as bracket width, torque angle, and wire size increased. Therefore, all bracket-archwire parameters except slot height had an effect on the critical contact angle. Results of the critical contact angle produced by our 3DMEs were the same as those produced by $3 \mathrm{D}$ CAD, confirming the validity of our derived equations. The results of this study provide a theoretic and experimental basis for clinical orthodontic practice and indicate that torque angles should be included in the evaluation of the critical contact angle.

\section{REFERENCES}

1. Tidy DC. Frictional forces in fixed appliances. Am J Orthod Dentofacial Orthop 1989;96:249-54.

2. Kusy RP, Whitley JQ. Assessment of second-order clearances between orthodontic archwires and bracket slots via the critical contact angle for binding. Angle Orthod 1999;69:71-80.

3. Kusy RP, Whitley JQ. Influence of archwire and bracket dimensions on sliding mechanics: derivations and determinations of the critical contact angles for binding. Eur J Orthod 1999;21:199208.

4. Frank CA, Nikolai RJ. A comparative study of frictional resistances between orthodontic bracket and arch wire. Am J Orthod 1980;78:593-609.

5. Angolkar PV, Kapila S, Duncanson MG Jr, Nanda RS. Evaluation of friction between ceramic brackets and orthodontic wires of four alloys. Am J Orthod Dentofacial Orthop 1990;98:499506.

6. Articolo LC, Kusy K, Saunders CR, Kusy RP. Influence of ceramic and stainless steel brackets on the notching of archwires during clinical treatment. Eur J Orthod 2000;22:409-25.

7. Kusy RP, Tobin EJ, Whitley JQ, Sioshansi P. Frictional coefficients of ion-implanted alumina against ion-implanted betatitanium in the low load, low velocity, single pass regime. Dent Mater 1992;8:167-72.

8. Dickson JA, Jones SP, Davies EH. A comparison of the frictional characteristics of five initial alignment wires and stainless steel brackets at three bracket to wire angulations - an in vitro study. Br J Orthod 1994;21:15-22.

9. Saunders CR, Kusy RP. Surface topography and frictional characteristics of ceramic brackets. Am J Orthod Dentofacial Orthop 1994;106:76-87.

10. Downing A, McCabe J, Gordon P. A study of frictional forces between orthodontic brackets and archwires. Br J Orthod 1994; 21:349-57.

11. Downing A, McCabe JF, Gordon PH. The effect of artificial saliva on the frictional forces between orthodontic brackets and archwires. Br J Orthod 1995;22:41-6.

12. Edwards GD, Davies EH, Jones SP. The ex vivo effect of ligation technique on the static frictional resistance of stainless steel brackets and archwires. Br J Orthod 1995 ;22:145-53.

13. Vaughan JL, Duncanson MG Jr, Nanda RS, Currier GF. Relative kinetic frictional forces between sintered stainless steel brackets and orthodontic wires. Am J Orthod Dentofacial Orthop 1995; 107:20-7.

14. Schlegel VJ. Relative friction minimization in fixed orthodontic bracket appliances. Biomech 1996;29:483-91.

15. Taylor NG, Ison K. Frictional resistance between orthodontic 
brackets and archwires in the buccal segments. Angle Orthod 1996;66:215-22.

16. Bazakidou E, Nanda RS, Duncanson MG Jr, Sinha P. Evaluation of frictional resistance in esthetic brackets. Am J Orthod Dentofacial Orthop 1997;112:138-44.

17. Kusy RP, Whitley JQ. Friction between different wire-bracket configurations and materials. Semin Orthod 1997;3:166-77.

18. Read-Ward GE, Jones SP, Davies EH. A comparison of selfligating and conventional orthodontic bracket systems. Br J Orthod 1997;24:309-17.

19. Pizzoni L, Ravnholt G, Melsen B. Frictional forces related to self-ligating brackets. Eur J Orthod 1998;20:283-91.

20. Braun S, Bluestein M, Moore BK, Benson G. Friction in perspective. Am J Orthod Dentofacial Orthop 1999;115:619-27.

21. Kapur R, Sinha PK, Nanda RS. Comparison of frictional resistance in titanium and stainless steel brackets. Am J Orthod Dentofacial Orthop 1999;116:271-4.

22. Loftus BP, Artun J, Nicholls JI, Alonzo TA, Stoner JA. Evaluation of friction during sliding tooth movement in various bracket-arch wire combinations. Am J Orthod Dentofacial Orthop 1999;116:336-45.

23. Michelberger DJ, Eadie RL, Faulkner MG, Glover KE, Prasad NG, Major PW. The friction and wear patterns of orthodontic brackets and archwires in the dry state. Am J Orthod Dentofacial Orthop 2000;118:662-74.

24. Zufall SW, Kusy RP. Sliding mechanics of coated composite wires and the development of an engineering model for binding. Angle Orthod 2000;70:34-47.

25. Articolo LC, Kusy RP. Influence of angulation on the resistance to sliding in fixed appliances. Am J Orthod Dentofacial Orthop 1999;115:39-51.

26. Meling TR, Ødegaard J, Meling EO. On mechanical properties of square and rectangular stainless steel wires tested in torsion. Am J Orthod Dentofacial Orthop 1997;111:310-20.

27. Kusy RP, O'Grady PW. Evaluation of titanium brackets for orthodontic treatment: Part II-the active configuration. Am J Orthod Dentofacial Orthop 2000;118:675-84.

28. Schumacher HA, Bourauel C, Drescher D. Frictional forces when rectangular guiding arches with varying edge bevel are employed. J Orofac Orthop 1998;59:139-49.

29. Meling TR, Ødegaard J. On the variability of cross-sectional dimensions and torsional properties of rectangular nickel-titanium arch wires. Am J Orthod Dentofacial Orthop 1998;113: 546-57.

30. Meling TR, Ødegaard J. The effect of cross-sectional dimensional variations of square and rectangular chrome-cobalt archwires on torsion. Angle Orthod 1998;68:239-48.

31. Fischer-Brandies H, Orthuber W, Es-Souni M, Meyer S. Torque transmission between square wire and bracket as a function of measurement, form and hardness parameters. J Orofac Orthop 2000;61:258-65.

\section{APPENDIX I}

With the y-axis as the axis of rotation (torque), the matrix has the form

$$
\left|\begin{array}{ccc}
\cos \phi & 0 & -\sin \phi \\
0 & 1 & 0 \\
\sin \phi & 0 & \cos \phi
\end{array}\right|
$$

The vectors after rotation around the y-axis are as follows:
The vector $A=<0,0, \mathrm{~A}>$ moves to the vector $A^{\prime}$ $=<-\mathrm{A} \sin \phi, 0, \mathrm{~A} \cos \phi>$,

the vector $B=<\mathrm{B}, 0,0>$ moves to the vector $B^{\prime}$ $=<\mathrm{B} \cos \phi, 0, \mathrm{~B} \sin \phi>$,

and the vector $L=<0, \mathrm{~L}, 0>$ moves to the vector $L^{\prime}=<0, \mathrm{~L}, 0>$.

Next, we calculated where the vectors $A^{\prime}, B^{\prime}$, and $L^{\prime}$ will be after rotation along the line from $O$ to $B^{\prime}$. The vector $B^{\prime}$ does not move with this action. Let $R$ be $B^{\prime}$, and suppose the vector $A^{\prime}$ goes to the vector $S=$ $<s_{1}, s_{2}, s_{3}>$, which satisfies the following conditions:

i) the angle between $A^{\prime}$ and $S$ is $\theta_{c}$;

ii) the length of the vector $S$ is $A$;

iii) the vector $S$ and $B^{\prime}$ is perpendicular, because $A^{\prime}$ and $B^{\prime}$ is so.

Because the inner product of 2 vectors is the product of the lengths of 2 vectors and $\cos \zeta$, where $\zeta$ is the angle between 2 vectors, we needed to know the angle between 2 vectors.

This was obtained from the condition i:

$$
A^{\prime} \cdot \mathrm{S}=\mathrm{A}^{2} \cos \theta_{\mathrm{c}}
$$

Therefore $\mathrm{A}^{\prime}$ goes to

$$
\begin{aligned}
\mathrm{S} & =<-\mathrm{A} \cos \theta_{\mathrm{c}} \sin \phi,-\mathrm{A} \sin \theta_{\mathrm{c}}, \mathrm{A} \cos \theta_{\mathrm{c}} \cos \phi>. \\
& =<-\mathrm{A} \sin \phi, 0, \mathrm{~A} \cos \phi>\cdot<0, \mathrm{~s}_{2}, \mathrm{~s}_{3}> \\
& =<-\mathrm{A} \mathrm{s}_{1} \sin \phi+\mathrm{A} \mathrm{s}_{3} \cos \phi>
\end{aligned}
$$

follows. Also, the square of the length of the vector $\mathrm{S}$, $|\mathrm{S}|^{2}=\mathrm{s}_{1}{ }^{2}+\mathrm{s}_{2}{ }^{2}+\mathrm{s}_{3}{ }^{2}=A^{2}$ follows from the condition ii.

Because the vector $\mathrm{S}$ is perpendicular to the vector $B^{\prime}$, the inner product of the vector $S$ and $B^{\prime}$,

$S \cdot B^{\prime}=0$

And

$$
\begin{aligned}
S \cdot B^{\prime} & =<\mathrm{s}_{1}, \mathrm{~s}_{2}, \mathrm{~s}_{3}>\cdot<\mathrm{B} \cos \phi, 0, \mathrm{~B} \sin \phi> \\
& =\mathrm{s}_{1} \mathrm{~B} \cos \phi+\mathrm{s}_{3} \mathrm{~B} \sin \phi=0 .
\end{aligned}
$$

Formula A2 gives this study $s_{1}=-s_{3} \tan \phi$ and after inserting it into formula A1, this study has

$$
\cos \theta_{c}=\frac{\left(s_{3} \cos \phi+s_{3} \tan \phi \sin \phi\right)}{A}
$$

or

$$
\cos \theta_{\mathrm{c}}=\frac{\mathrm{s}_{3}\left(\cos ^{2} \phi+\sin ^{2} \phi\right)}{\mathrm{A} \cos \phi}=\frac{\mathrm{s}_{3}}{\mathrm{~A} \cos \phi}
$$

Multiplying $\mathrm{A} \cos \phi$ on both sides, $\mathrm{s}_{3}=\mathrm{A} \cos \theta_{\mathrm{c}} \sin \phi$ comes out. 
Because $\mathrm{s}_{1}=-\mathrm{s}_{3} \tan \phi$ and $|S|^{2}=s_{1}{ }^{2}+s_{2}{ }^{2}+$ $s_{3}^{2}=A^{2}, s_{1}=-\mathrm{A} \cos \theta_{\mathrm{c}} \sin \phi$ and $s_{2}= \pm \mathrm{A} \sin \theta_{\mathrm{c}}$ (this study can ignore addition sign).

Therefore $A^{\prime}$ goes to $S=<-\mathrm{A} \cos \theta_{\mathrm{c}} \sin \phi,-\mathrm{A}$ $\sin \theta_{\mathrm{c}}, \mathrm{A} \cos \theta_{\mathrm{c}} \cos \phi>$.

On the other hand, the vector

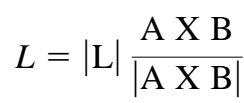

for the vector $\mathrm{L}$ is perpendicular to the vectors $A$ and $B$.

Because the rotation action preserves the angles, the vector $L^{\prime}=L$ goes to the vector

$$
|\mathrm{L}| \frac{\mathrm{S} \times \mathrm{B}^{\prime}}{\left|\mathrm{S} \times \mathrm{B}^{\prime}\right|}
$$

say the vector $T$ and the vector $T=<-\mathrm{L} \sin \theta_{\mathrm{c}} \sin \phi$, $\mathrm{L} \cos \theta_{\mathrm{c}}, \mathrm{L} \sin \theta_{\mathrm{c}} \cos \phi>$.

As shown in Figure 2, the parallelepiped was determined by the vectors $R, S$, and $T$ as the wire. We simply needed to cut the wire, allowing it to fit in the bracket. The parallelepiped was cut with the xz-plane, the plane $\mathrm{y}=$ bracket width $b$ and the plane $\mathrm{z}=$ bracket size $a$ to fit it in the bracket. Then the common point of parallelepiped, $\mathrm{y}=b$ and $\mathrm{z}=a$, has the $b$ as the second coordinate and $a$ as the third coordinate. The common point is the end point of the vector $R+S+T$.

Hence the following 2 conditions are obtained:

i) $-\mathrm{A} \sin \theta_{\mathrm{c}}+\mathrm{L} \cos \theta_{\mathrm{c}}=b$

ii) $\mathrm{A} \cos \theta_{\mathrm{c}} \cos \phi+\mathrm{B} \sin \phi+\mathrm{L} \sin \theta_{\mathrm{c}} \cos \phi=a$

$\mathrm{L}$ will be omitted, and we next determine $\cos \theta_{\mathrm{c}}$.

$\theta_{c}$ is the critical contact angle after torque that we wish to obtain.

The condition i) gives

$$
\mathrm{L}=\frac{\mathrm{b}+\mathrm{A} \sin \theta_{\mathrm{c}}}{\cos \theta_{\mathrm{c}}}
$$

After inserting it in the condition ii) and this study has $\mathrm{A} \cos \theta_{\mathrm{c}} \cos \phi+\mathrm{B} \sin \phi$

$$
+\frac{\left(b+A \sin \theta_{c}\right) \sin \theta_{\mathrm{c}} \cos \phi}{\cos \theta_{\mathrm{c}}}=\mathrm{a}
$$

or

$\mathrm{A} \cos \phi+\mathrm{B} \cos \theta_{\mathrm{c}} \sin \phi+b \sin \theta_{\mathrm{c}} \cos \phi=a \cos \theta_{\mathrm{c}}$

or $b \sin \theta \cos \phi=(a-\mathrm{B} \sin \phi) \cos \theta-\mathrm{A} \cos \phi$

or

$$
\begin{aligned}
& b^{2}\left(1-\cos ^{2} \theta_{c}\right) \cos ^{2} \phi=(a-B \sin \phi)^{2} \cos ^{2} \theta \\
& -2 A \cos \phi(a-B \sin \phi)+A^{2} \cos ^{2} \phi
\end{aligned}
$$

or

$(a-B \sin \phi)^{2}+b^{2} \cos \phi^{2} \cos \theta^{2}-2 \mathrm{~A} \cos \phi$

$$
\cdot(a-B \sin \phi) \cos \theta_{\mathrm{c}}+\left(\mathrm{A}^{2}-b^{2}\right) \cos ^{2} \phi=0 .
$$

Solving this second-order equation (Equ. 3), $a$ is bracket slot size, $b$ is bracket width, $A$ is archwire height, $B$ is archwire width, $\theta_{c}$ is critical contact angle, and $\phi$, torque angle.

This formula is the derived 3DME for the critical contact angle.

\section{APPENDIX II}

In the case of torque $\phi=0$, we were able to replicate formula A3. The critical contact angle $\theta_{c}$ decreases as the torque $\phi$ increases. We next looked at which torque $\phi$ results in a critical contact angle of 0 . This was determined from the previous 2 conditions when $\theta=0$. In other words, $\cos \theta=1$ and $\sin \theta=0$.

After putting $\theta=0$,

$$
\begin{aligned}
(a-\mathrm{B} \sin \phi)^{2}-2 \mathrm{~A} \cos \phi(a-\mathrm{B} & \sin \phi) \\
& +\mathrm{A}^{2} \cos ^{2} \phi=0 .
\end{aligned}
$$

or

$$
a-(\mathrm{B} \sin \phi-\mathrm{A} \cos \phi)^{2}=0 .
$$

or

$$
a=\mathrm{B} \sin \phi+\mathrm{A} \cos \phi .
$$

or

$$
\left(\mathrm{A}^{2}+\mathrm{B}^{2}\right) \cos ^{2} \phi-2 a \mathrm{~A} \cos \phi+\left(a^{2}-\mathrm{B}^{2}\right)=0 .
$$

or

$$
\cos \phi=\frac{a^{2}-B^{2}}{\sqrt{A a \pm \sqrt{ } B^{2}\left(-a^{2}+A^{2}+B^{2}\right)}}
$$

$a$ is bracket slot size, $A$ is archwire height, $B$ is archwire width, and $\phi$ is torque angle.

This formula (A4) is the maximum torque angle with the given archwire and orthodontic bracket.

Equ. 3.

$$
\cos \theta_{\mathrm{c}}=\frac{\mathrm{A}^{2} \cos ^{2} \phi-\mathrm{b}^{2} \cos ^{2} \phi}{\mathrm{A} \cos \phi(\mathrm{a}-\mathrm{B} \sin \phi) \pm \sqrt{\mathrm{b}^{2} \cos ^{2} \phi\left(-(\mathrm{A} \cos \phi)^{2}+(\mathrm{a}+\mathrm{B} \sin \phi)^{2}+(\mathrm{b} \cos \phi)^{2}\right.}}
$$

